\title{
Follicle-stimulating Hormone Is Secreted More Irregularly Than Luteinizing Hormone in Both Humans and Sheep
}

\author{
Steven M. Pincus, ${ }^{\star}$ Vasantha Padmanabhan, ${ }^{\ddagger}$ William Lemon, ${ }^{\ddagger}$ John Randolph, ${ }^{\ddagger}$ and A. Rees Midgley ${ }^{\ddagger}$ \\ *Guilford, Connecticut 06437; and ${ }^{\ddagger}$ Reproductive Sciences Program, University of Michigan, Ann Arbor, Michigan 48109
}

\begin{abstract}
Recently introduced statistical tools capable of discerning differences between the pattern of luteinizing hormone ( $\mathrm{LH})$ secretion and that of follicle-stimulating hormone (FSH) could be valuable in understanding ovulation and menopause, and ultimately in making diagnostic decisions and treating infertility and polycystic ovary syndrome. We assessed the validity and scope of the hypothesis that FSH is secreted more irregularly than LH in ewes and fertile women. We compared secretory irregularity of $\mathrm{LH}$ to that of FSH in both ovariectomized ewes $(n=7)$ and women of proven fertility $(n=5)$ during the follicular and luteal phases of their reproductive cycles. In each sheep, time series from both hypophyseal portal blood (HPB) and peripheral blood were evaluated in 72 samples obtained every 5 min; in each human, both luteal and follicular periods were studied in 192 samples obtained every $7.5 \mathrm{~min}$. To quantify serial irregularity, we used approximate entropy (ApEn), a scale- and model-independent statistic. FSH secretion was consistently more irregular than that of $\mathrm{LH}$ in each subject. For sheep HPB, ApEn(FSH) $=1.415 \pm 0.097$ was larger than $\operatorname{ApEn}(\mathrm{LH})=0.822 \pm 0.213, P<0.0001($ mean $\pm \mathrm{SD}$, paired $t$ test). This difference persisted peripherally: $\operatorname{ApEn}\left(\mathrm{FSH}_{\mathrm{per}}\right)=$ $1.431 \pm 0.101>\operatorname{ApEn}\left(\mathrm{LH}_{\mathrm{per}}\right)=1.252 \pm 0.086, P=0.024$. In women, $\operatorname{ApEn}(\mathrm{FSH})=1.467 \pm 0.217>\operatorname{ApEn}(\mathrm{LH})=0.923 \pm$ 0.305, $P<0.0001$. ApEn(FSH) $>\operatorname{ApEn}(\mathrm{LH})$ in $100 \%$ of women (peripheral) and sheep HPB. Secretion during the follicular phase was more irregular than during the luteal phase for both FSH and LH $(P<0.01)$. LH mean level secretion showed a wake/sleep difference in women, $P<$ 0.005 , with higher values awake. The consistency and statistical significance of these findings suggest that this LH/FSH difference may be broadly based within higher mammals. Ranges of normative and abnormal regularity values of $\mathrm{LH}$, FSH, and their difference can be used in a number of settings, both (currently) research and (potentially ultimately) clinical milieus. (J. Clin. Invest. 1998. 101:1318-1324.) Key words: gonadotropin • irregularity $\bullet$ pulsatility $\bullet$ menopause • infertility
\end{abstract}

Portions of this work were presented at the 24th annual meeting of the Society of Neuroscience in Miami, FL, 13-18 November 1994 and at the 10th International Congress of Endocrinology in San Francisco, CA, 12-15 June 1996.

Address correspondence to Steven M. Pincus, 990 Moose Hill Road, Guilford, CT 06437. Phone: 203-458-3455; FAX: 203-458-9063.

Received for publication 20 June 1997 and accepted in revised form 30 December 1997.

J. Clin. Invest.

(C) The American Society for Clinical Investigation, Inc. 0021-9738/98/03/1318/07 \$2.00

Volume 101, Number 6, March 1998, 1318-1324

http://www.jci.org

\section{Introduction}

At least $15 \%$ of married couples in the USA ( $\sim 4.5$ million) of reproductive age will experience some degree of infertility (1). Infertility difficulties are enhanced with increasing female age: among healthy women without detectable reproductive disorders, infertility in women ages $35-39$ is estimated to be $\sim 25 \%$, and in women $40 \mathrm{yr}$ or older, $\sim 27 \%$ (1). The properly regulated release of hormones by both the hypothalamus and pituitary gland is one essential requirement for monthly ovulation. If the hypothalamus functions abnormally, follicles will develop improperly, often resulting in anovulation. Thus, greater understanding of gonadotropin-releasing hormone $(\mathrm{GnRH}){ }^{1}$ luteinizing hormone $(\mathrm{LH})$, and follicle-stimulating hormone (FSH) secretion, and concomitant relationships among these hormones, is of vital importance scientifically, in understanding the physiology of ovulation, and ultimately clinically in treating disorders.

Until recently, word models of pituitary secretion of $\mathrm{LH}$ and FSH rendered their dynamics in a virtually identical fashion since they are presumed to be driven commonly by $\mathrm{GnRH}$ (2). Assuming common stimulation has led to tacit discounting of differences in their secretory patterns or attribution of those differences to changes or differences in clearance rates or isoforms $(2,3)$. Recently, detailed characterizations of circulating patterns of LH and FSH have uncovered differences in regulation of these two hormones, giving credence to efforts to measure and understand differences in patterns of secretion (4-6). Visually, it appears that the secretory pattern of FSH in sheep is more complicated than that of LH, with many more minor episodes or subordinate activity when measured from hypophyseal portal blood (HPB), and somewhat less so when measured in peripheral blood (Fig. 1) (7). Motivated by this observation, we attempted to quantify the observed differences using approximate entropy (ApEn) $(8,9)$ (described below), determine the breadth of the apparent finding across species by analyses of both ovariectomized ewes and humans, and estimate the consistency of the finding.

\section{Methods}

Sheep study. The experimental design and description of assays of LH and FSH in peripheral (jugular) and HPB of ovariectomized sheep used in this study, herein statistically analyzed by ApEn, have been described previously $(7,10,11)$. This method for monitoring $\mathrm{LH}$ and FSH in portal blood reveals the dynamics of pituitary hormone secretion without deconvolution (11). In brief, the procedure involved surgically fitting ovariectomized Suffolk ewes with an apparatus for collection of HPB $(12,13) .1$ wk later, the sheep were heparin-

1. Abbreviations used in this paper: ApEn, approximate entropy; $\mathrm{FSH}$, follicle-stimulating hormone; $\mathrm{GH}$, growth hormone; GnRH, gonadotropin-releasing hormone; HPB, hypophyseal portal blood; LH, luteinizing hormone. 

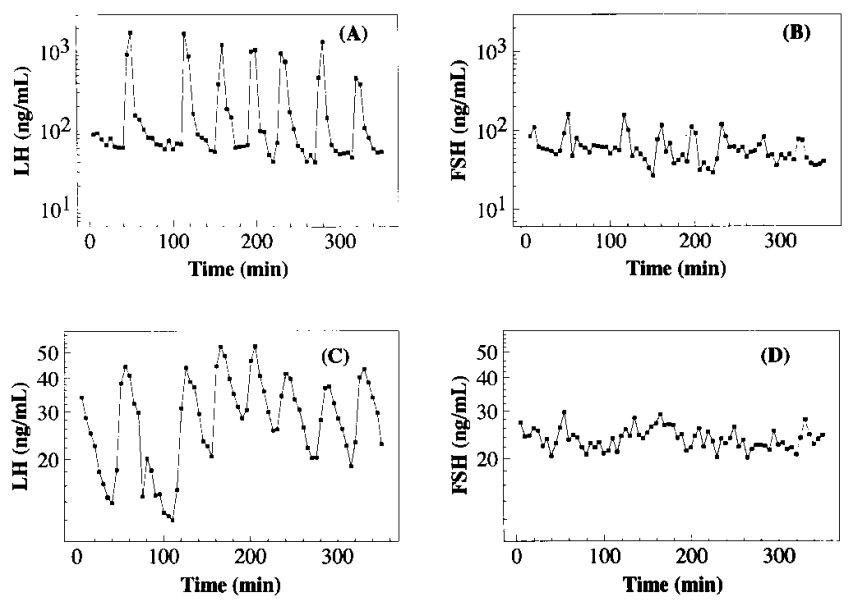

Figure 1. LH and FSH concentrations ( $\mathrm{ng} / \mathrm{ml})$ measured at 5-min intervals for $6 \mathrm{~h}$ from an ovariectomized ewe, with respective ApEn $(1,20 \% \mathrm{SD})$ values. $(A)$ Secreted $(\mathrm{HPB}) \mathrm{LH}, \mathrm{ApEn}=0.625 ;(B) \mathrm{se}-$ creted $(\mathrm{HPB}) \mathrm{FSH}, \mathrm{ApEn}=1.256 ;(C)$ peripheral LH, ApEn $=$ 1.161 ; and $(D)$ peripheral FSH, ApEn $=1.486$.

ized, the anterior face of the pituitary was lesioned, catheters from the apparatus and jugular vein were run to fraction collectors in an adjoining room, and portal and peripheral blood were collected separately as 5-min integrated samples. Portal blood was collected in tubes with $0.5 \mathrm{ml}$ of $0.3 \mathrm{mM}$ bacitracin in saline and later adjusted for dilution. In this study, $n=7$ sets of 72 samples of plasma obtained during the breeding season over $6 \mathrm{~h}$ (10) were analyzed. Concentrations of ovine LH (14) and ovine FSH (15-17) were measured in all series. All procedures were done with the approval of the University's Committee on Use and Care of Animals (UCUCA) in accordance with NIH guidelines.

Human study. Five women of proven fertility were studied (ages 18-35). Proven fertility was established by the requirement that the subjects were pregnant within the previous 48 mo. Exclusion criteria included concurrent medical illness or psychiatric problems, the use of oral contraceptives or any medication known, or with the potential, to interfere with the hormonal axes under study during the past 6 mo, or use of any medication other than for a cold or pain during the past $4 \mathrm{mo}$. Volunteers had to be nonsmokers with regular nighttime sleep habits who had regular menstrual cycles (27-31 d). All subjects gave informed consent. Samples $(2.5 \mathrm{ml})$ of peripheral blood were withdrawn every $7.5 \mathrm{~min}$ for $24 \mathrm{~h}$, providing an opportunity to assess diurnal variation. Sleep and wake periods were recorded every $7.5 \mathrm{~min}$. Blood was drawn with a dual stopcock technique using a discard syringe at the distal stopcock and, to keep the line open, a slow intravenous infusion of $0.9 \mathrm{~N}$ saline at the proximal stopcock. After drawing, tubes of blood samples were kept at room temperature for at most $2 \mathrm{~h}$ and then refrigerated until separation. Each subject was studied twice, once during the early follicular phase (days 2-5) and, after one intervening cycle, during the midluteal phase (days 16-23). Samples were analyzed for human LH and human FSH by automated, solid phase, magnetically separated, competitive, two site, acridinium ester chemiluminescence-based immunometric assays on a Ciba-Corning Diagnostics analyzer (ACS 180; Medfield, MA).

Quantification of episodicity. To quantify irregularity, we use ApEn, a model-independent statistic (for definition see reference 8), with further mathematical properties and representative biological applications $(9,18-22)$. ApEn is complementary to pulse detection algorithms widely used to evaluate hormone secretion time series (23). ApEn evaluates both dominant and subordinate patterns in data; notably, it will detect changes in underlying episodic behavior that do not reflect in peak occurrences or amplitudes (24), a point that is ger- mane to this analysis. Additionally, ApEn provides a direct barometer of feedback system change in many coupled systems $(24,25)$. In endocrine pathophysiology, ApEn has shown vivid distinctions $(P<$ $10^{-10}$ ) between normal and tumor-bearing subjects for growth hormone (GH) (26), ACTH and cortisol (27), and aldosterone (28), a pronounced and consistent gender difference in GH irregularity in both humans and rats (29), and a positive correlation between greater irregularity (larger ApEn) of GH and advancing age (30).

ApEn assigns a nonnegative number to a time series, with larger values corresponding to greater apparent process randomness (serial irregularity), and smaller values corresponding to more instances of recognizable features or patterns in the data. Two input parameters, $m$ and $r$, must be specified to compute ApEn. ApEn measures the logarithmic likelihood that runs of patterns that are close (within $r$ ) for $m$ contiguous observations remain close (within the same tolerance width $r$ ) on next incremental comparisons (8).

For this study, we calculated ApEn values for all data sets, $m=1$ and $r=20 \%$ of the standard deviation (SD) of the individual subject time series. Normalizing $r$ to each time series SD gives ApEn a translation- and scale-invariance to absolute serum concentration levels (19). ApEn is a relative measure of process regularity, and can show significant variation in its absolute numerical value with changing background noise characteristics. Since ApEn generally increases with increasing process noise (and increasing intraassay variation), it is appropriate to compare data sets with similar assay CVs, as we do here. Also, most importantly, despite algorithmic similarities, $\operatorname{ApEn}(m, r)$ is not intended $(8,9,31)$ as an approximate value of the Kolmogorov-Sinai (K-S) entropy. It is essential to consider $\operatorname{ApEn}(m, r)$ as a family of parameters; comparisons are intended with fixed $m$ and $r$.

Previous studies that included both theoretical analysis $(8,31,32)$ and clinical applications (18-22, 26-30) have demonstrated that the input parameters indicated above produce good statistical validity (reproducibility) for ApEn for time series of the lengths considered herein ( $n \geq 60$ data points). Specifically, via extensive Monte Carlo calculations, the standard deviation of $\operatorname{ApEn}(m=1, r=20 \% \mathrm{SD}, n) \leq$ 0.06 for length- $n$ sequences, $n \geq 60$, for a large class of diverse candidate models, including low-order autoregressive moving average models, the composite oscillator-noise family of processes MIX(p) (for definition see reference 8), and parameterized families of deterministic, dynamical systems. The ApEn application with $m=1$ estimates the rate of entropy for a first-order $(m=1)$ approximating Markov Chain to the underlying true process (33). Further technical discussion of mathematical and statistical properties of ApEn, including robustness to noise and artifacts, mesh interplay, relative consistency of $(m, r)$ pair choices, asymptotic normality under general assumptions, statistical bias, and error estimation for general processes can be found elsewhere $(31,32)$. To develop a more intuitive, physiological understanding of the ApEn definition, a multistep description of its typical algorithmic implementation, with figures, is available (32).

Wake/sleep distinction. In the human study, we tested to determine the presence of a wake/sleep distinction, comparing mean (absolute) levels from each state for both LH and FSH. Given a significant difference between states, noted below, it became imperative to select a similar, homogeneous subepoch of the 24-h studies for each subject, to avoid confounding interpretation (induced by nonstationarities) of subsequent statistical analyses. For this purpose, we analyzed a contiguous block of awake-state data for all subjects: the final $12 \mathrm{~h}$ of study for all but one data set; for subject 4, follicular state, the final 9.5-h epoch, 10:30 a.m. to 8 p.m., to avoid a brief sleep period 8 a.m. to $10: 30$ a.m.

Statistical analysis. All statistical comparisons below for discrimination between two groups used the paired $t$ test with unknown variance. Both raw data time series and logarithmically transformed time series were analyzed; the latter analysis is complementary to the analysis of the raw data, as it diminishes the effects of very large peaks, implicitly weighs more heavily the dynamics occurring at low secretory levels, and reduces the heteroscedasticity in the data. 


\section{Results}

\section{Sheep study}

Representative sheep LH and FSH concentration time series derived from previously published studies $(7,10,11)$ are shown in Fig. 1, $A$ and $B$ for HPB and $C$ and $D$ for peripheral blood; individual subject ApEn values for all time series are given in Table I. Group comparisons of ApEn for secreted HPB FSH (1.415 \pm 0.097$)$ and HPB LH $(0.822 \pm 0.213)$ differed $(P=0.000077)$ (paired $t$ test). As noted in Table I, pairwise $\mathrm{ApEn}(\mathrm{FSH})$ was much larger (more irregular) than ApEn(LH) for each ewe. Importantly, this difference persisted in peripheral blood, with a group mean ApEn for FSH $(1.431 \pm 0.101)$ larger than that for LH $(1.252 \pm 0.086)(P=0.024)$. Furthermore, perhaps because of dispersion, dilution, and elimination, sampling peripherally gives a much more irregular signal, e.g., mean ApEn for peripheral LH is larger than that for HPB LH $(P=0.00033)$.

Analyses of the logarithmically transformed time series were qualitatively very similar: group comparisons of ApEn for secreted HPB FSH (1.472 \pm 0.087$)$ and HPB LH (1.140土 $0.128)$ differed $(P=0.000011)$. Pairwise, $\mathrm{ApEn}(\mathrm{FSH})$ was again much larger than ApEn(LH) for each ewe. In peripheral data, mean ApEn for FSH (1.447 \pm 0.072$)$ was larger than that for LH $(1.272 \pm 0.079)(P=0.012)$.

\section{Human study}

Wake/sleep comparison. For each subject and phase, for each of LH and FSH, we compared mean secretion levels from 1 a.m. to 7 a.m. (subjects asleep for $>90 \%$ of the time) to those from 8 a.m. to 8 p.m. (subjects awake for $>95 \%$ of the time). The mean LH concentration was lower during the nocturnal period $(3.12 \pm 2.30 \mathrm{mIU} / \mathrm{ml})$ than during the diurnal period $(4.31 \pm 2.05$ $\mathrm{mIU} / \mathrm{ml})(P<0.03)$, confirming the visually suggested state difference seen in Fig. 2. For the logarithmically transformed time series, the mean $(\log ) \mathrm{LH}$ concentration was lower during the nocturnal period $(0.80 \pm 0.67 \mathrm{mIU} / \mathrm{ml})$ than during the diurnal period $(1.31 \pm 0.38 \mathrm{mIU} / \mathrm{ml})(P<0.005)$. Concomitantly, the mean nocturnal $\mathrm{LH}$ was smaller than mean diurnal $\mathrm{LH}$ for 9 of the 10 subjects both for the untransformed and log-transformed data. The corresponding FSH comparisons approached significance: mean nocturnal FSH was lower (3.82 \pm $1.40 \mathrm{mIU} / \mathrm{ml})$ than diurnal FSH $(4.22 \pm 1.35 \mathrm{mIU} / \mathrm{ml})(P<0.07)$; and log-transformed nocturnal FSH was lower $(1.27 \pm 0.36$ $\mathrm{mIU} / \mathrm{ml})$ than diurnal FSH $(1.39 \pm 0.31 \mathrm{mIU} / \mathrm{ml})(P<0.07)$. As noted above, we accordingly performed subsequent statistical analysis on a contiguous block of awake-state data.

Table I. Individual Subject LH and FSH ApEn Values for Ewes

\begin{tabular}{lcccc}
\hline Subject & $\begin{array}{c}\text { LH } \\
\text { secreted (HPB) }\end{array}$ & $\begin{array}{c}\text { FSH } \\
\text { secreted (HPB) }\end{array}$ & $\begin{array}{c}\text { LH } \\
\text { peripheral }\end{array}$ & $\begin{array}{c}\text { FSH } \\
\text { peripheral }\end{array}$ \\
\hline Ewe 1 & 0.758 & 1.396 & 1.299 & 1.440 \\
Ewe 2 & 0.903 & 1.505 & 1.242 & 1.561 \\
Ewe 3 & 0.625 & 1.256 & 1.161 & 1.486 \\
Ewe 4 & 0.610 & 1.347 & 1.315 & 1.400 \\
Ewe 5 & 0.696 & 1.487 & 1.235 & 1.252 \\
Ewe 6 & 1.199 & 1.527 & 1.379 & 1.372 \\
Ewe 7 & 0.963 & 1.385 & 1.133 & 1.505 \\
\hline
\end{tabular}

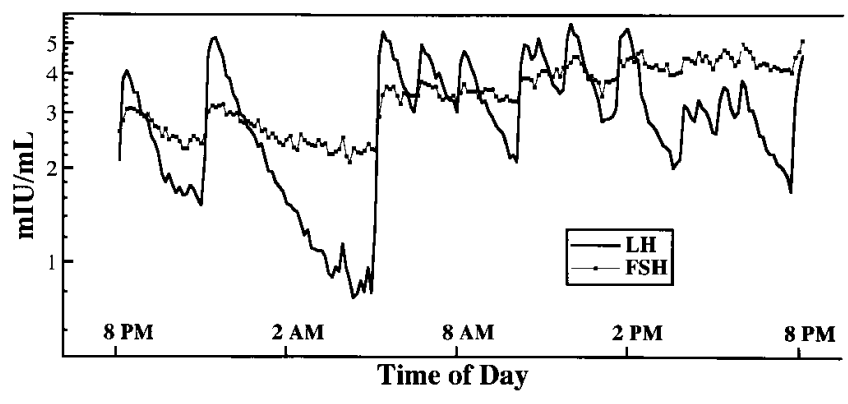

Figure 2. 24-h LH and FSH serum concentrations (mIU/ml) measured at 7.5-min intervals from a human female, who slept from 11:30 p.m. to 5 a.m. Observe an apparent decrease in LH, and to a somewhat lesser extent, in FSH, during the night.

Stationary epoch, final $12 \mathrm{~h}$. Representative human $\mathrm{LH}$ and FSH peripheral blood time series are shown in Fig. 3, $A$ and $B$ (follicular phase) and $C$ and $D$ (luteal phase); individual subject mean levels and ApEn values for all time series are given in Table II. First, pooling luteal and follicular analyses, group comparisons $(n=10)$ of $\mathrm{ApEn}(\mathrm{FSH})(1.467 \pm 0.217)$ and ApEn(LH) $(0.923 \pm 0.305)$ differed $(P=0.000016)$. Pairwise, $\operatorname{ApEn}(\mathrm{FSH})$ was again much larger than $\operatorname{ApEn}(\mathrm{LH})$ for each subject and cycle stage. In each of the luteal and follicular stages, ApEn distinctions were significant: in the follicular stage, $\operatorname{ApEn}(\mathrm{FSH})(1.591 \pm 0.126)$ and $\mathrm{ApEn}(\mathrm{LH})(1.136 \pm 0.259)$ differed $(P=0.0052)$. ApEn(FSH) $(1.343 \pm 0.227)$ and ApEn(LH) $(0.710 \pm 0.170)$ also differed in the luteal phase $(P=0.0024)$.

Furthermore, there was a follicular/luteal phase distinction, for each of $\mathrm{LH}$ and FSH. For LH, follicular ApEn (1.136 \pm $0.259)$ was larger than luteal ApEn $(0.710 \pm 0.170)(P=$

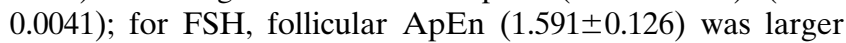
than luteal ApEn (1.343 \pm 0.227$)(P=0.0094)$.
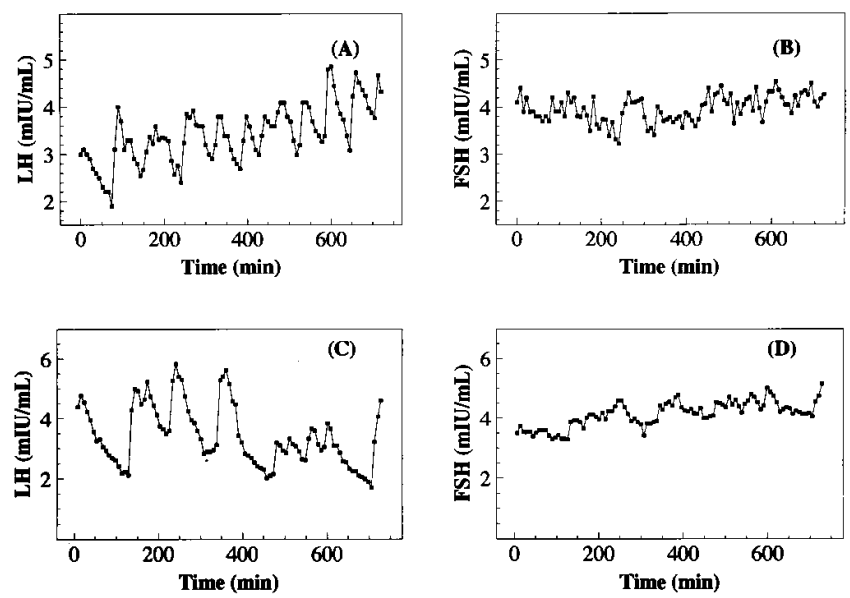

Figure 3. Serum LH and FSH concentrations (mIU/ml) for 12 awake $\mathrm{h}$, measured at 7.5-min intervals from a human female, with respective mean and ApEn (1,20\% SD) values. Early follicular phase: $(A)$ $\mathrm{LH}$, mean $=3.43, \mathrm{ApEn}=1.215 ;(B) \mathrm{FSH}$, mean $=3.96, \mathrm{ApEn}=$ 1.568. Luteal phase: $(C) \mathrm{LH}$, mean $=3.42, \mathrm{ApEn}=0.911 ;(D) \mathrm{FSH}$, mean $=4.13, \mathrm{ApEn}=1.230$. Note apparent differences in irregularity between corresponding FSH and LH series, for each phase, and between follicular and luteal series, for each of FSH and LH, quantified by ApEn. 
Table II. Individual Subject LH and FSH Mean and ApEn Values in Human Females

\begin{tabular}{lccccc}
\hline & \multicolumn{2}{c}{ Mean } & & \multicolumn{2}{c}{ ApEn } \\
\cline { 2 - 3 } \cline { 5 - 6 } File name & LH & FSH & & LH & FSH \\
\hline 1: Follic. & 2.779 & 6.995 & & 0.755 & 1.494 \\
1: Luteal & 2.400 & 4.089 & & 0.535 & 1.095 \\
2: Follic. & 3.258 & 5.218 & & 1.307 & 1.780 \\
2: Luteal & 3.730 & 2.815 & & 0.790 & 1.657 \\
3: Follic. & 3.429 & 3.958 & 1.215 & 1.568 \\
3: Luteal & 3.418 & 4.129 & 0.911 & 1.230 \\
4: Follic. & 5.221 & 3.521 & 1.001 & 1.469 \\
4: Luteal & 9.454 & 3.304 & 0.529 & 1.237 \\
5: Follic. & 5.432 & 5.554 & 1.402 & 1.646 \\
5: Luteal & 3.978 & 2.620 & 0.786 & 1.495 \\
& & & &
\end{tabular}

Contiguous wakeful epoch occurred during the final $12 \mathrm{~h}$, except in subject 4 , follicular phase, when it occurred during the final $9.5 \mathrm{~h}$.

As above, analyses of the logarithmically transformed time series were very similar: Pooled $(n=10)$ luteal/follicular group comparisons of ApEn(FSH) (1.464 \pm 0.217$)$ and ApEn(LH) $(0.964 \pm 0.235)$ differed $\left(P<10^{-5}\right)$. Pairwise, ApEn(FSH) was again much larger than $\mathrm{ApEn}(\mathrm{LH})$ for each subject and cycle stage. In the follicular stage, ApEn(FSH) $(1.579 \pm 0.096)$ and $\operatorname{ApEn}(\mathrm{LH})(1.114 \pm 0.229)$ differed $(P=0.0025)$, while in the

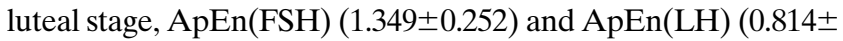
$0.127)$ differed $(P=0.0031)$. The follicular/luteal phase distinction persisted: for $\mathrm{LH}$, follicular ApEn was larger than luteal ApEn $(P=0.0081)$; for FSH, follicular ApEn was larger than luteal ApEn $(P=0.03)$.

Notably, there was no difference in mean LH $(4.31 \pm 2.05$ $\mathrm{mIU} / \mathrm{ml})$ and FSH $(4.22 \pm 1.35 \mathrm{mIU} / \mathrm{ml})$ levels $(P>0.9)$. Similarly, for the log-transformed data, mean LH $(1.31 \pm 0.38 \mathrm{mIU} /$ $\mathrm{ml})$ and $\mathrm{FSH}(1.39 \pm 0.31 \mathrm{mIU} / \mathrm{ml})$ were not significantly different $(P>0.9)$.

\section{Discussion}

Summarizing the statistical results, several distinct analyses confirm that FSH secretion is consistently and significantly more irregular than LH secretion, suggesting that this difference may be broadly based. In the women, these distinctions were consistent for each of the luteal and follicular phases. Mean LH and FSH secretion values were indistinct in women, reinforcing the utility of the distinct, complementary perspective taken by an assessment of irregularity. The luteal phase/ follicular phase distinction is concordant with the slower luteal pulse generation. Finally, the day/night secretory differences seen here reconfirm previously established findings for both LH and, to a lesser extent, FSH (34-36).

Complementarity of irregularity and pulsatility analyses. As noted above, ApEn is complementary to pulse detection algorithms widely used in the evaluation of hormone secretion time series, with the nature of this complementarity discussed previously (24). While in many settings pulse identification methods are quite sufficient to characterize and distinguish distinct physiologic states, in many other settings, it seems that there is a need for added analysis beyond monitoring the pulse count and related statistics. For example, brief, irregular oscillations in plasma insulin levels in maturity-onset diabetics (37), in $\mathrm{GH}$ secretion time series of acromegalics and acromegalics in remission (26), in GH time series for healthy female rats (29), and in testosterone time series in men, especially older men $(38,39)$, clear pulse identification appears to be a very challenging endeavor.

In such instances where clear pulse identification is difficult, the practical utility of irregularity assessment is that there is frequently a gain in statistical clarity and reproducibility by applying ApEn, compared to algorithms that first require pulse determinations. Two examples of this are in direct comparisons of ApEn to pulsatility method findings on common data sets: studies $(38,39)$ of changes in $\mathrm{LH}$ and testosterone in younger versus older males, and studies $(40,41)$ of changes in FSH in younger versus older females. In both pairs of studies, the ApEn analyses yielded clearer statistical significance between groups, greater sensitivity and specificity, and afforded qualitatively new significant inferences, compared to those derived from pulse analysis. Descriptively, ApEn thus provides a robust measure of the extent and persistence of distinct levels and types of both primary and subordinate activity, without needing to identify discrete pulses in the process.

The above issues are manifested in the present context of analysis of LH/FSH hormonal dynamics in two different ways. First, the identification and characterization of discrete FSH pulses, especially in peripheral blood, has proved to be a formidable endeavor. The particular difficulties with the assessment of episodic FSH release have been reviewed in some detail $(7,41)$, and relate in great part to the slow metabolic clearance of the hormone. This reinforces the need for techniques to statistically assess a degree of more subtle structure in FSH serial dynamics, apart from a requirement to do pulse identification first.

Second, even in HPB, assessing the true secretory dynamics, based on and by combining pulse analysis for sheep $(7,11)$, we infer a nearly 1:1 correspondence between GnRH, LH, and primary FSH pulses. Namely, we note a virtually $100 \%$ association between GnRH and LH pulses (11), and a 93\% concordance of GnRH pulses with essentially coincident, clearly discrete FSH pulses in the portal plasma (7). Yet, there is considerably more subordinate FSH than LH activity in HPB, which one wishes to quantitatively assess by suitable analytical methods (7).

Furthermore, in this study, for the HPB sheep data, we determined that for each of $\mathrm{LH}$ and $\mathrm{FSH}$, there was insignificant linear correlation between ApEn values and mean (primary pulse) frequency and amplitude levels, per sheep. This near independence of ApEn and pulsatility assessment in sheep portal blood empirically reinforces the complementarity of these distinct approaches.

Finally, the utility of quantitatively assessing the FSH/LH irregularity difference, and changes thereto, is seen in a parallel set of studies (41). Three groups of healthy human subjects were studied: $n=24$ younger females ( 8 per menstrual cycle stage, 3 cycle stages), $n=8$ postmenopausal females, and $n=17$ males, across a broad spectrum of ages, 21-79 y. LH and FSH (peripheral) serum samples were obtained every $10 \mathrm{~min}$ for $24 \mathrm{~h}$. First, this study (41) reconfirms the above young women $\mathrm{FSH} /$ LH irregularity difference, via a different study design and sampling regimen. For young females, FSH was consistently 
more irregular than LH, per subject $(P<0.00001)$, with $\operatorname{ApEn}(\mathrm{FSH})>\operatorname{ApEn}(\mathrm{LH})$ for 23 of 24 subjects. Importantly, and a primary finding of this study, for the postmenopausal women, the irregularity difference vanished, ApEn(FSH) $\operatorname{ApEn}(\mathrm{LH})=0.008 \pm 0.205$. Interestingly, males exhibited qualitatively similar results: $\mathrm{ApEn}(\mathrm{FSH})-\mathrm{ApEn}(\mathrm{LH})$ was significantly and negatively correlated with age $(r=-0.75$, $P=0.0006)$. The above observation that clear FSH pulse identification in peripheral blood is especially challenging is confirmed visually (41), particularly for older subjects.

Differential regulation of $\mathrm{LH}$ and FSH. While many studies have described physiologic stage-specific changes in trains of episodic pulses of circulating LH, relatively few studies have focused on FSH or the relationship of these hormones. These two glycoprotein hormones are known to be contained in the same pituitary cell (42), the gonadotrope, stored in granules and secreted in response to GnRH. Existing literature concerning the differential regulation of $\mathrm{LH}$ and FSH has emphasized changes in mean concentrations and their response to physiologic perturbations. Detailed description of the secretory character of FSH, the regularity of $\mathrm{LH}$ and FSH, and the regularity of their differences, has not been ascertained previously.

Because GnRH can release both hormones from the same cell and because a separate FSH-releasing hormone has not been isolated, the prevailing concept has been that GnRH serves as the principal secretagogue for both hormones (2). According to this notion, observed differences in the ratio of peripheral concentration of the two hormones (43) result in part from the impact of endocrine, paracrine, and autocrine effectors on the gonadotrope that cause differential changes in synthesis or storage of the two hormones (3). GnRH stimulation could then lead to differences in the quality and, thus, circulatory half-life of each hormone or in the relative amounts of their secretion (3). While the two hormones are known to have different metabolic clearance rates (3), with the half-life of FSH greatly exceeding $\mathrm{LH}$, and while different physiologic states can lead to changes in the ratios of different FSH isoforms, these differences do not appear to account for the fairly abrupt changes in the LH/FSH ratios in the circulation (44). Additional explanations for observed differences have included the possible role of gonadotrope subpopulations with differential response (45) or response times (46) to GnRH.

Included among the endocrine effectors with potential for differential regulation of $\mathrm{LH}$ and FSH is the mode of presentation of GnRH. Long interpulse duration (47) and low concentration infusions of GnRH (48) have been associated with enhanced release of FSH in vivo while shorter interpulse intervals have been reported to increase LH (47). However, changes in the mode by which GnRH is introduced in vitro have not led to substantial changes in the differential secretion of LH and FSH from pituitary cells (49). Other endocrine, paracrine, or autocrine effectors include estradiol, inhibin, activin, follistatin, neulopeptide $\mathrm{Y}$, and galanin. A detailed review of the putative and demonstrated roles of these agents is beyond the scope of this discussion. Suffice it to note that they have been reported to act on gonadotropes to stimulate or inhibit the transcription or translation of FSH $\beta$ mRNA (50) or otherwise affect synthesis and secretion of both gonadotropins. Some of the agents appear to act directly, others appear to act by modulating the response to $\operatorname{GnRH}(51,52)$, perhaps by affecting the availability of GnRH receptor.
In contrast to these attempts to account for differences in observed $\mathrm{LH} / \mathrm{FSH}$ ratios through modifications of the effects of $\mathrm{GnRH}$ as the primary secretagogue, other evidence points to the existence of at least one GnRH-independent mechanism controlling FSH secretion. Thus, following hypothalamicpituitary disconnection in sheep, FSH but not LH, continues to be secreted for long periods (53). FSH secretion also continues in hypophysectomized rats when pituitary glands are transplanted beneath their kidney capsules (4) and FSH secretion by cultures of pituitary glands continues for long periods of time (54). Other evidence includes the observation of selective secretion of FSH but loss of LH in intact animals passively neutralized with antiserum to $\operatorname{GnRH}(5,55)$. In the sheep pituitary secretion data analyzed above, subordinate elevations of FSH are observed between primary LH pulses, beyond concomitant primary LH and FSH pulses. Possibly most convincingly, administration of a GnRH antagonist wiped out all GnRH-dependent LH and FSH pulses (measured directly at the face of the pituitary), but left quasipulsatile, basal secretion of FSH largely intact (6).

Statistically, the ApEn analysis herein also is consistent with a more complicated control process for FSH than for LH. Elsewhere, we have analytically linked ApEn to a mechanistic understanding via mathematical network analysis, for general classes of networks $(24,25)$. In particular, we demonstrated that ApEn increases with greater system coupling and/or greater external influences, e.g., the number of critical interacting factors, in many coupled, complicated systems, including autoregressive moving average and graded superposition models, and coupled stochastic differential equations. Thus the increase observed above in $\mathrm{ApEn}(\mathrm{FSH})$ compared to $\operatorname{ApEn}(\mathrm{LH})$ should be anticipated if, as suggested by many investigators (7), FSH is controlled by $\mathrm{GnRH}$ and the putative FSH-releasing factor, while LH is controlled just by GnRH. Therefore, ApEn quantifies the subordinate FSH elevations that qualitatively produce more apparently complicated, irregular behavior for FSH than for LH while simultaneously allowing some broadly held mechanistic inferences to be made.

Potential applications. These findings suggest that characterization of regularity of both $\mathrm{LH}$ and $\mathrm{FSH}$, as well as their mutual relationship, could be used in a number of contexts. Of course, until a simple, reliable, unobtrusive, safe, and inexpensive method is available for continuous or episodic measurement, this kind of analysis cannot be used routinely in clinical settings. To date, frequent sampling has only been used in research settings studying normal or abnormal physiology; the cost of many samples and the inconvenience and discomfort to patients will maintain that practice, in the near term. But the improvement in understanding of a number of abnormal states, e.g., polycystic ovary syndrome, premature ovarian failure, and ovulatory infertility, may lead to improvements in FSH therapy or in alternative therapies targeted at FSH release and based on new findings regarding the differential control of gonadotropin secretion.

Among clinically oriented studies, it would seem worthwhile to investigate if regularity characteristics differ in polycystic ovary syndrome, and how infertility drugs such as clomiphene citrate affect LH and FSH signal regularity, both individually and jointly. Also, with recent technologic advances, controlled synthetic FSH production is now available, allowing greater control in the LH/FSH administration in infertility therapy. Statistical means of assessing an optimal LH/ 
FSH mixture are clearly desirable, which the above analytic strategy (and results) can begin to address. Finally, the distinction in irregularity between luteal and follicular phases, for both LH and FSH, may be useful not only in possibly predicting an LH surge, but may be consequential in determining the time until onset of menopause, often an important determinant in hormonal therapy versus surgical (hysterectomy) clinical options in a patient with perimenopausal menorrhagia. We hypothesize that as women become perimenopausal, luteal/ follicular ApEn differences diminish, for both LH and FSH.

\section{Acknowledgments}

The authors would like to acknowledge the efforts of Geoffrey E. Dahl, Neil P. Evans, Douglas L. Foster, Judy M. Manning, Kimberly P. Mayfield, and Sue M. Moenter in collecting the samples analyzed in this study and the effort of Karen Nairn in handling the recruitment and scheduling of the volunteers. The authors appreciate the assistance of the nursing staff of the General Clinical Research Center of the University of Michigan in obtaining the frequent samples of blood from the women.

This work was supported in part by the National Institute of Child Health and Human Development grant R43 HD-33776, and performed in part for the National Institute of Child Health and Human Development's National Cooperative Program on Infertility Research (National Institutes of Health grant U54 HD-29184), using samples generated in an earlier study funded by the National Institutes of Health grant R01 HD-18018, receiving the support of the Assay and Reagents, Sheep Research, and Biostatistics Cores of the Center for the Study of Reproduction (National Institutes of Health grant P30 HD-18258) and the Clinical Research Center (National Institutes of Health grant MO1 RR-00042) at the University of Michigan.

\section{References}

1. Mosher, W.D. 1988. Fecundity and infertility in the United States. Am. J. Public Health. 78:181-182.

2. Schally, A.V., A. Arimura, A.J. Kastin, H. Matsuo, Y. Baba, T.W. Redding, R.M. Nair, L. Debeljuk, and W.F. White. 1971. Gonadotropin-releasing hormone: one polypeptide regulates secretion of luteinizing and follicle-stimulating hormones. Science. 173:1036-1038.

3. Ulloa-Aguirre, A., A.R. Midgley, Jr., I.Z. Beitins, and V. Padmanabhan. 1995. Follicle stimulating isohormones: biological characterization and physiological relevance. Endocr. Rev. 16:765-787.

4. DePaolo, L.V. 1991. Hypersecretion of follicle-stimulating hormone (FSH) after ovariectomy of hypophysectomized, pituitary-grafted rats: implications for local regulatory control of FSH. Endocrinology. 128:1731-1740.

5. McCann, S.M., H. Mizunuma, W.K. Samson, and M.D. Lumpkin. 1983. Differential hypothalamic control of FSH secretion: a review. Psychoneuroendocrinology. 8:299-308.

6. Padmanabhan, V., K.L. McFadden, N.P. Evans, G.E. Dahl, D.T. Mauger, and F.J. Karsch. 1994. Pulsatile FSH secretion in the ovariectomized ewe is controlled by both GnRH-dependent and GnRH-independent mechanisms. Abstr. Soc. Neurosci. 20:436.6a. (Abstr.)

7. Padmanabhan, V., K. McFadden, D.T. Mauger, F.J. Karsch, and A.R. Midgley, Jr. 1997. Neuroendocrine control of FSH secretion. I. Direct evidence for separate episodic and basal components of FSH secretion. Endocrinology. 138:424-432.

8. Pincus, S.M. 1991. Approximate entropy as a measure of system complexity. Proc. Natl. Acad. Sci. USA. 88:2297-2301.

9. Pincus, S., and B.H. Singer. 1996. Randomness and degrees of irregularity. Proc. Natl. Acad. Sci. USA. 93:2083-2088.

10. Karsch, F.J., G.E. Dahl, N.P. Evans, J.M. Manning, K.P. Mayfield, S.M. Moenter, and D.L. Foster. 1993. Seasonal changes in gonadotropin-releasing hormone secretion in the ewe: alteration in response to the negative feedback action of estradiol. Biol. Reprod. 49:1377-1383.

11. Midgley, A.R., Jr., K. McFadden, M. Ghazzi, F.J. Karsch, M.B. Brown, D.T. Mauger, and V. Padmanabhan. 1997. Nonclassical secretory dynamics of LH revealed by hypothalamo-hypophyseal portal sampling of sheep. Endocrine. 6:133-143.

12. Clarke, I.J., and J.T. Cummins. 1982. The temporal relationship between gonadotrophin-releasing hormone $(\mathrm{GnRH})$ and luteinizing hormone
(LH) secretion in ovariectomized ewes. Endocrinology. 111:1737-1739.

13. Caraty, A., A. Locatelli, S.M. Moenter, and F.J. Karsch. 1994. Sampling of hypophyseal portal blood of conscious sheep for direct monitoring of hypothalamic neurosecretory substances. In Pulsatility in Neuroendocrine Systems. J.E. Levine and P.M. Conn, editors. Academic Press, New York. 162-183.

14. Niswender, G.D., A.R. Midgley, Jr., S.E. Monroe, and L.E. Reichert, Jr 1968. Radioimmunoassay for rat luteinizing hormone with antiovine LH serum and ovine LH-131-I. Proc. Soc. Exp. Biol. Med. 128:807-811.

15. L'Hermite, M., G.D. Niswender, L.E. Reichert, Jr., and A.R. Midgley, Jr. 1972. Serum follicle-stimulating hormone in sheep as measured by radioimmunoassay. Biol. Reprod. 6:325-332.

16. Padmanabhan, V., K.M. Reno, M. Borondy, T.D. Landefeld, F.J. Ebling, D.L. Foster, and I.Z. Beitins. 1992. Effect of nutritional repletion on pituitary and serum follicle-stimulating hormone isoform distribution in growthretarded lambs. Biol. Reprod. 46:964-971.

17. Padmanabhan, V., C.D. Mieher, M. Borondy, H. I'Anson, R.I. Wood, T.D. Landefeld, D.L. Foster, and I.Z. Beitins. 1992. Circulating bioactive follicle-stimulating hormone and less acidic follicle-stimulating hormone isoforms increase during experimental induction of puberty in female lambs. Endocrinology. 131:213-220.

18. Kaplan, D.T., M.I. Furman, S.M. Pincus, S.M. Ryan, L.A. Lipsitz, and A.L. Goldberger. 1991. Aging and the complexity of cardiovascular dynamics. Biophys. J. 59:945-949.

19. Pincus, S.M., T.R. Cummins, and G.G. Haddad. 1993. Heart rate control in normal and aborted SIDS infants. Am. J. Physiol. 264:R638-R646.

20. Pincus, S.M., I.M. Gladstone, and R.A. Ehrenkranz. 1991. A regularity statistic for medical data analysis. J. Clin. Monit. 7:335-345.

21. Pincus, S.M., and R.R. Viscarello. 1992. Approximate entropy: a regularity measure for fetal heart rate analysis. Obstet. Gynecol. 79:249-255.

22. Ryan, S.M., A.L. Goldberger, S.M. Pincus, J. Mietus, and L.A. Lipsitz. 1994. Gender- and age-related differences in heart rate dynamics: are women more complex than men? J. Am. Coll. Cardiol. 24:1700-1707.

23. Urban, R.J., W.S. Evans, A.D. Rogol, D.L. Kaiser, M.L. Johnson, and J.D. Veldhuis. 1988. Contemporary aspects of discrete peak-detection algorithms. I. The paradigm of the luteinizing hormone pulse signal in men. Endocr. Rev. 9:3-37.

24. Pincus, S.M., and D.L. Keefe. 1992. Quantification of hormone pulsatility via an approximate entropy algorithm. Am. J. Physiol. 262:E741-E754.

25. Pincus, S.M. 1994. Greater signal regularity may indicate increased system isolation. Math. Biosci. 122:161-181.

26. Hartman, M.L., S.M. Pincus, M.L. Johnson, D.H. Matthews, L.M. Faunt, M.L. Vance, M.O. Thorner, and J.D. Veldhuis. 1994. Enhanced basal and disorderly growth hormone $(\mathrm{GH})$ secretion distinguish acromegalic from normal pulsatile GH release. J. Clin. Invest. 94:1277-1288.

27. Van den Berg, G., S.M. Pincus, J.D. Veldhuis, M. Frolich, and F. Roelfsema. 1997. Greater disorderlines of ACTH and cortisol release accompanies pituitary-dependent Cushing's disease. Eur. J. Endocrinol. 136:394-400.

28. Siragy, H.M., W.V.R. Vieweg, S.M. Pincus, and J.D. Veldhuis. 1995. Increased disorderliness and amplified basal and pulsatile aldosterone secretion in patients with primary aldosteronism. J. Clin. Endocrinol. Metab. 80:28-34.

29. Pincus, S.M., E. Gevers, I.C.A.F. Robinson, G. van den Berg, F. Roelfsema, M.L. Hartman, and J.D. Veldhuis. 1996. Females secrete growth hormone with more process irregularity than males in both humans and rats. Am. J. Physiol. 270:E107-E115.

30. Veldhuis, J.D., A.Y. Liem, S. South, A. Weltman, J. Weltman, D.A. Clemmons, R. Abbott, T. Mulligan, M.L. Johnson, S. Pincus, et al. 1995. Differential impact of age, sex steroid hormones, and obesity on basal versus pulsatile growth hormone secretion in men as assessed in an ultrasensitive chemiluminescence assay. J. Clin. Endocrinol. Metab. 80:3209-3222.

31. Pincus, S.M., and W.M. Huang. 1992. Approximate entropy: statistical properties and applications. Commun. Statist. Theory Meth. 21:3061-3077.

32. Pincus, S.M., and A.L. Goldberger. 1994. Physiological time series analysis: what does regularity quantify? Am. J. Physiol. 266:H1643-H1656.

33. Pincus, S.M. 1992. Approximating Markov chains. Proc. Natl. Acad. Sci. USA. 89:4432-4436.

34. Rossmanith, W.G., and S.S.C. Yen. 1987. Sleep-associated decrease in luteinizing hormone pulse frequency during the early follicular phase of the menstrual cycle: evidence for an opiodergic mechanism. J. Clin. Endocrinol. Metab. 65:715-718.

35. Venturoli, S., E. Porcu, R. Fabbri, O. Magrini, L. Gammi, R. Paradisi, M. Forcacci, R. Bolzani, and C. Flamigni. 1988. Episodic pulsatile secretion of $\mathrm{FSH}, \mathrm{LH}$, prolactin, oestradiol, oestrone, and LH circadian variations in polycystic ovary syndrome. Clin. Endocrinol. 28:93-107.

36. O'Byrne, K.T. J.C. Thalabard, P.M. Grosser, R.C. Wilson, C.L. Williams, M.D. Chen, D. Ladendorf, J. Hotchkiss, and E. Knobil. 1991. Radiotelemetric monitoring of hypothalamic gonadotropin-releasing hormone pulse generator activity throughout the menstrual cycle of the rhesus monkey. Endocrinology. 129:1207-1214.

37. Lang, D.A., D.R. Matthews, and R.C. Turner. 1981. Brief, irregular oscillations of basal plasma insulin and glucose concentrations in diabetic men Diabetes. 30:435-439.

38. Mulligan, T., A. Iranmanesh, S. Gheorghiu, M. Godschalk, and J.D. 
Veldhuis. 1995. Amplified nocturnal luteinizing hormone (LH) secretory burst frequency with selective attenuation of pulsatile (but not basal) testosterone secretion in healthy aged men: possible Leydig cell desensitization to endogenous LH signaling - a clinical research center study. J. Clin. Endocrinol. Metab. 80: 3025-3031.

39. Pincus, S.M., T. Mulligan, A. Iranmanesh, S. Gheorghiu, M. Godschalk, and J.D. Veldhuis. 1996. Older males secrete luteinizing hormone (LH) and testosterone more irregularly, and jointly more asynchronously, than younger males. Proc. Natl. Acad. Sci. USA. 93:14100-14105.

40. Booth, R.A., Jr., J.Y. Weltman, V.I. Yankov, J. Murray, T.S. Davison, A.D. Rogol, C.M. Asplin, M.L. Johnson, J.D. Veldhuis, and W.S. Evans. 1996. Mode of pulsatile FSH secretion in gonadal-hormone sufficient and deficient women. J. Clin. Endocrinol. Metab. 81:3208-3214.

41. Pincus, S.M., J.D. Veldhuis, T. Mulligan, A. Iranmanesh, and W.S. Evans. 1997. Effects of age on the irregularity of LH and FSH serum concentrations in women and men. Am. J. Physiol. (Endocrinol Metab.). 273:E989-E995.

42. Childs, G.V., C. Hyde, Z. Naor, and K. Catt. 1983. Heterogeneous luteinizing hormone and follicle-stimulating hormone storage patterns in subtypes of gonadotropes separated by centrifugal elutriation. Endocrinology. 113: $2120-2128$.

43. Chappel, S.C. 1985. Neuroendocrine regulation of luteinizing hormone and follicle-stimulating hormone: a review. Life Sci. 36:97-103.

44. Levine, J.E., and M.T. Duffy. 1988. Simultaneous measurement of luteinizing hormone $(\mathrm{LH})$-releasing hormone, $\mathrm{LH}$, and follicle-stimulating hormone release in intact and short-term castrate rats. Endocrinology. 122:22112221.

45. Denef, C., E. Hautekeete, and R. Dewals. 1978. Monolayer cultures of gonadotrophs separated by velocity sedimentation: heterogeneity in response to luteinizing hormone-releasing hormone. Endocrinology. 103:736-747.

46. Schwartz, N.B., C. Rivier, J. Rivier, and W.W. Vale. 1985. Effect of gonadotropin-releasing hormone antagonists on serum follicle-stimulating hormone and luteinizing hormone under conditions of singular follicle-stimulating hormone secretion. Biol. Reprod. 32:391-398.

47. Clarke, I.J., J.T. Cummins, J.K. Findlay, K.J. Burman, and B.W. Doughton. 1984. Effects on plasma luteinizing hormone and follicle-stimulating hormone of varying the frequency and amplitude of gonadotropin-releasing hormone pulses in ovariectomized ewes with hypothalamo-pituitary disconnection. Neuroendocrinology. 39:214-221.

48. Wise, P.M., N. Rance, G.D. Barr, and C.A. Barraclough. 1979. Further evidence that luteinizing hormone-releasing hormone also is follicle-stimulating hormone-releasing hormone. Endocrinology. 104:940-947.

49. Kotsuji, F., S.J. Winters, H.S. Keeping, B. Attardi, H. Oshima, and P. Troen. 1988. Effects of inhibin from primate Sertoli cells on follicle-stimulating hormone and luteinizing hormone release by perifused rat pituitary cells. Endocrinology. 122:2796-2802.

50. McNeilly, A.S. 1988. The control of FSH secretion. Acta Endocrinol. Suppl. (Copenh.). 288:31-40.

51. Landefeld, T.D., T. Bagnell, and I. Levitan. 1989. Effects of estradiol on gonadotropin subunit messenger ribonucleic acid amounts during an induced gonadotropin surge in anestrous ewes. Mol. Endocrinol. 3:10-14.

52. Wu, J.C., S.C. Sealfon, and W.L. Miller. 1994. Gonadal hormones and gonadotropin-releasing hormone $(\mathrm{GnRH})$ alter messenger ribonucleic acid levels for GnRH receptors in sheep. Endocrinology. 134:1846-1850.

53. Hamernik, D.L., and T.M. Nett. 1988. Gonadotropin-releasing hormone increases the amount of messenger ribonucleic acid for gonadotropins in ovariectomized ewes after hypothalamic-pituitary disconnection. Endocrinology. 122:959-966.

54. Sheridan, R., B. Loras, L. Surardt, F. Ectors, and J.L. Pasteels. 1979. Autonomous secretion of follicle-stimulating hormone by long term organ cultures of rat pituitaries. Endocrinology. 104:198-204.

55. Culler, M.D., and A. Negro-Vilar. 1987. Pulsatile follicle-stimulating hormone secretion is independent of luteinizing hormone-releasing hormone (LHRH): pulsatile replacement of LHRH bioactivity in LHRH-immunoneutralized rats. Endocrinology. 120:2011-2021. 